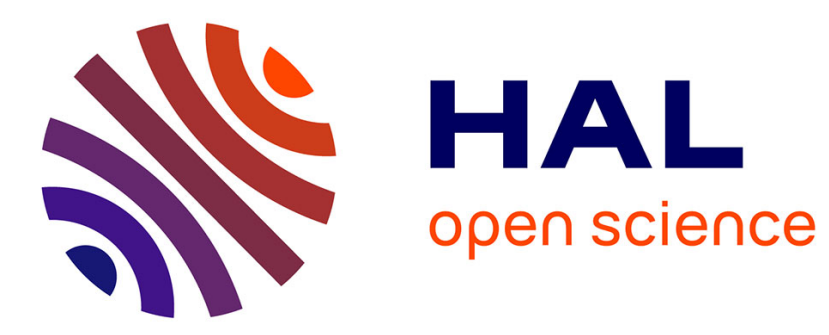

\title{
Parametric Identification of a Powered Two-Wheeled Vehicles: Algebraic Approach
}

Majda Fouka, Pierre-Marie Damon, Lamri Nehaoua, Hichem Arioui, Saïd Mammar

\section{- To cite this version:}

Majda Fouka, Pierre-Marie Damon, Lamri Nehaoua, Hichem Arioui, Saïd Mammar. Parametric Identification of a Powered Two-Wheeled Vehicles: Algebraic Approach. 2017. hal-01520798

\section{HAL Id: hal-01520798 \\ https://hal.science/hal-01520798}

Preprint submitted on 16 May 2017

HAL is a multi-disciplinary open access archive for the deposit and dissemination of scientific research documents, whether they are published or not. The documents may come from teaching and research institutions in France or abroad, or from public or private research centers.
L'archive ouverte pluridisciplinaire HAL, est destinée au dépôt et à la diffusion de documents scientifiques de niveau recherche, publiés ou non, émanant des établissements d'enseignement et de recherche français ou étrangers, des laboratoires publics ou privés. 


\title{
Parametric Identification of a Powered Two-Wheeled Vehicles : Algebraic Approach
}

\author{
M. Fouka, P-M. Damon, L. Nehaoua, H. Arioui and S. Mammar
}

\begin{abstract}
The paper aims to identify model's parameters of powered two-wheeled vehicles (PTWv) allowing us to simulate and interpret its lateral dynamics. The motorcycle motion is identified in order to conduct a preliminary study of simulated behavior of the vehicle while riding.

An algebraic identification method for continuous-time linear system is used to obtain an accurate model of the motorcycle under the steering inputs persistent condition, vehicle dynamics tools are mainly used to simulate the different responses and to compare the identified parameters using the BikeSim software.

The validation of this model is investigated using data measurement in order to confirm the accuracy of the parameters estimation.
\end{abstract}

\section{INTRODUCTION}

Powered two-wheeled vehicles (PTWv) are an increasingly popular means of transport, especially for the opportunities it offers to avoid congestion. But the PTWv still remains to this day, a particularly dangerous transportation mode. Studies on powered two-wheelers have historically been few [1] [2]. This lack of studies raises the question of the relevance of the solutions adopted to reduce accidents.

The objective of our research is to study motorcycle turning, the development of active riding assistances and preventive safety systems that can warn riders upstream of hazardous driving situations to ensure acceptable levels of comfort and safety, taking into account the infrastructure and the near environment [3] [4].

In this perspective, measures aiming to reduce vulnerable riders accidents should be studied, taking into account the fact that PTWv have a specific dynamic behavior that will sometimes lead to greater control difficulties [5].

The purpose of this paper is to present the particularities of a motorcycle and the characteristic variables of the vehicle dynamics. A simple model is considered to describe the lateral behavior of the yaw and roll motions. So as to understand the dynamics of the vehicle and the excitations to which it is subject.

The idea of using identification from measurements comes naturally: to determine the physical parameters necessary for the simulation based on the tests and measurements carried out on the vehicle. It is then possible, by calculating the dynamic model of the system, to build a simulation model and to identify the parameters. To the knowledge of the author, works on the parametric identification of the PTWv have been very few, main research were achieved without considering the physical model of the two-wheeled

All the authors are with the IBISC Laboratory, Evry-Val-d'Essonne University, 40, rue de Pelvoux, 91020 Evry Courcouronne Cedex majda.foukadens.univ-evry. fr vehicle. In [6], the author considers an autoregressive model of motorcycle to estimate the state space model of lateral dynamics without identifying parameters. There are other research axes which are concerned with the identification of the controller parameters to stabilize the motorcycle, in the book [7], authors explore the field of the black box control-oriented modeling, by presenting a case study of direct identification from experiment. In [8], authors study the handling of a motorcycle out of plane dynamics with a robust MBD model to ensure directional stability and identify the key parameters.

An algebraic identification method is used to obtain an accurate model of the real system. The algebraic estimation method presented by Fliess and al. in [9] is used for continuous-time linear system. The algebraic method has already been applied with good results in a wide range of applications, like: flexible robots estimation, Mass-SpringDamper model, Identification Method for a DC Motor ([10],[11],[12],[14]). The advantages of this approach are that the method does not require initial conditions and requires dependence between the system (input / output) and the algorithm is computed on-line (Instantaneous convergence). The difficulties encountered concern the choice of the persistent input excitation in real situation.

In this paper the identification algorithm has been verified by simulation, the validation of this model will be investigated with the use of data carried out on the PTWv ; The paper is organized as follows. Section II briefly describes the PTWv's lateral dynamics. Section III presents a static method to identify geometric parameters and an algebraic method to identify inertial parameters of the model. Finally, section V provides some simulation results.

\section{TWO-WHEELED LATERAL DYNAMICS DESCRIPTION}

\section{A. Geometry and the Motorcycle Description}

In the present work, the motorcycle is considered as a rigid body. The movements of the motorcycle are characterized

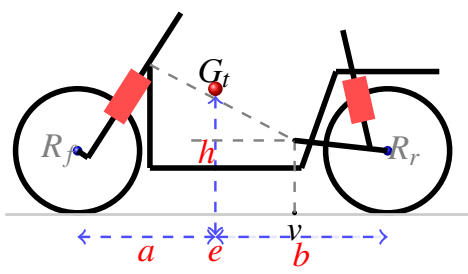

Fig. 1. The motorcycle geometrical description 
by three translations: the longitudinal, lateral and vertical position $(x, y, z)$, and three rotations: the roll $\phi$ about the longitudinal axis $x$, the pitch $\theta$ about the transverse axis $y$ and the yaw $\psi$ about the vertical axis $z$.

The inertial parameters of the motorcycle are generally represented by: it mass $m$, the moments of inertia : $I_{x}, I_{y}, I_{z}$ and the position of its center of gravity $G_{t}$, for more detail please refer to [13].

One can note that these parameters are unknown.

\section{B. Dynamic Model of a Rigid Motorcycle}

In this part, the lateral movement of the powered twowheeled vehicle is considered by simplifying the sharp model [1], with the assumption that the roll axis is considered on the ground.

Let us consider the following motorcycle model with 3 DoF giving the lateral, the yaw and the roll motion dynamics:

$$
\left\{\begin{aligned}
m\left(v_{y}+\dot{\psi} v_{x}\right) & =F_{y f}+F_{y r} \\
I_{z} \ddot{\psi} & =a F_{y f}-b F_{y r} \\
I_{x} \ddot{\phi}-m h\left(v_{y}+\dot{\psi} v_{x}\right) & =m g h \phi
\end{aligned}\right.
$$

and the tire relaxation dynamics

$$
\left\{\begin{array}{c}
F_{y f}=-\frac{v_{x}}{\tau} F_{y f}+C_{f 1}\left(\delta-\frac{v_{y}-a \dot{\psi}}{v_{x}}\right)+C_{f 2} \phi+\Delta F_{y f}^{S} \\
\dot{F_{y r}}=-\frac{v_{x}}{\tau} F_{y r}-C_{r 1}\left(\frac{v_{y}-b \dot{\psi}}{v_{x}}\right)+C_{r 2} \phi+\Delta F_{y r}^{S}
\end{array}\right.
$$

where $\Delta F_{y r}^{S}, \Delta F_{y f}^{S}$ are the nonlinear parts of forces which characterizes the dangerous situations (saturation of the forces) and $\tau$ is a scalar defining the length of relaxation, all the variables are listed in appendix.

These equations allow us to describe the lateral dynamics of a motorcycle modeled as a single rigid body.

Let $x(t)=\left(\begin{array}{llllll}v_{y} & \dot{\psi} & \phi & \dot{\phi} & F_{y f} & F_{y r}\end{array}\right)^{T}$, adding the trivial expressions $\dot{\phi}=\dot{\phi}$. We deduce the following Linear Parameter Varying (LPV) system :

$$
E \dot{x}(t)=M\left(v_{x}(t)\right) x(t)+N u(t)+F\left(v_{x}(t)\right) d(t)
$$

where the input $u(t)$ is the sterring angle $\delta$ and $d(t)$ is the nonlinear part of the lateral forces. Measurable values are the roll and yaw rates $\dot{\psi}, \dot{\phi}$ and $a_{y}=\frac{1}{m}\left(F_{y f}+F_{y r}\right)$. Then the output equation can be written $y(t)=C x(t)$.

Please refer to Appendix for the expressions of matrix $E$, $M, N, F$ and $C$.

Since the matrix $E$ is non-singular, then the system can be transformed in the following form

$$
\dot{x}(t)=A\left(v_{x}(t)\right) x(t)+B u(t)+Q\left(v_{x}(t)\right) d(t)
$$

with

$$
\left\{\begin{aligned}
A\left(v_{x}(t)\right) & =E^{-1} M\left(v_{x}(t)\right) \\
B & =E^{-1} N \\
Q\left(v_{x}(t)\right) & =E^{-1} F\left(v_{x}(t)\right)
\end{aligned}\right.
$$

Finally, the single-body motorcycle model is described as an LPV model.

$$
\left\{\begin{array}{l}
\dot{x}(t)=A\left(v_{x}(t)\right) x(t)+B u(t)+Q\left(v_{x}(t)\right) d(t) \\
y(t)=C x(t)
\end{array}\right.
$$

\section{IDENTIFICATION OF THE MOTORCYCLE DYNAMIC}

\section{A. Identification of $a, b$ and $h$ parameters by a static method}

The position of the center of gravity $G_{t}$ of the motorcycle has a significant influence on the lateral dynamics. In this part, we endeavor to describe a practical method by applying the static (force and moment) fundamental principle, and with the use of simple tools to record the values of the vertical forces on the front and rear wheels.

The center of mass for a single-body model of a motorcycle is defined by the values of the geometric parameters $a, b$ and $h$.

let's consider that the motorcycle is positioned on a slope (figure 2),

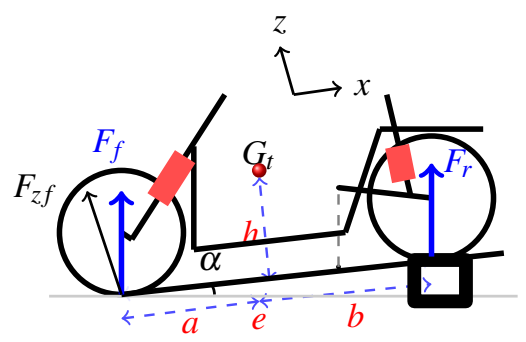

Fig. 2. The balance of forces - motorcycle on the slope in the opposite direction (1st Case).

Where,

- $F_{z f}$ (resp. $F_{z r}$ ): the component of the vertical force applied to the front wheel (or rear wheel, measured with a weight person scale) along the $\mathrm{Z}$ axis.

- $P_{x}=P \sin (\alpha)$ : the component of the weight along the $\mathrm{X}$-axis.

- $\alpha$ : the angle of the slope.

- $P=m g$ : represents the force of gravity applied to the center of mass.

Now, we consider the "Inclined" configuration to seek the "horizontal" position of $G_{t}(a$ and $b$ ) and the "vertical" position $(h)$. The motorcycle is lifted up with a hydraulic lifting goat to a desired angle of inclination $\alpha$, we put a wedge under the rear wheel, and the person scale under the front wheel (1st case : (figure 2)), the same procedure is performed by turning the motorcycle in the reverse configuration, (2nd case). The angle of the slope $\alpha$ can easily be calculated by trigonometry.

The equations of forces and moments in projection on the $x$ and $z$ axis allow us to find $a, b$ and $h$ at the same time:

$$
\left\{\begin{aligned}
F_{f z_{1}}+F_{r z_{1}}=F_{f z_{2}}+F_{r z_{2}} & =P \\
P \tan (\alpha) h+F_{f z_{1}} a-F_{r z_{1}} b & =0 \text { (1st case) } \\
P \tan (\alpha) h-F_{f z_{2}} a+F_{r z_{2}} b & =0 \text { (2nd case) } \\
a+b & =e
\end{aligned}\right.
$$

The motorcycle position can be located by solving the set of equations, we get for $\mathrm{a}, \mathrm{b}$ and $\mathrm{h}$ :

$$
\left(\begin{array}{l}
a \\
b \\
h
\end{array}\right)=\left(\begin{array}{ccc}
F_{f z_{1}} & -F_{r z_{1}} & P \cdot \tan (\alpha) \\
-F_{f z_{2}} & F_{r z_{2}} & P \cdot \tan (\alpha) \\
1 & 1 & 0
\end{array}\right)^{-1} \times\left(\begin{array}{l}
0 \\
0 \\
e
\end{array}\right)
$$




\section{B. Algebraic identification approach}

The goal of this section is the parameters identification of a time invariant linear system modeled by a rational transfer function by knowing the output signal $y(t)$ and the input signal $u(t)$, we attempts to estimate the parameters of the model, the advantages are that the estimation does not require initial conditions or dependence between the system input and output and the algorithm is computed on-line. [15],[9] [16].

\section{Identifiability:}

The parameters are linearly identifiable if, and only if,[9]

$$
\mathscr{P} \times \Theta^{T}=\mathscr{Q}
$$

where

- $\mathscr{P}$ and $\mathscr{Q}$ are respectively $(8 \times 8)$ and $(8 \times 1)$ matrices.

- $\Theta$ is the set of parameters.

- $\operatorname{det}(\mathscr{P}) \neq 0$.

The algebraic approach is mainly based on the robust computation of the time-derivatives of signal by using a finite weighted combination of time-integrations of this signal. These results allow to obtain an estimate of the timederivative of a particular order in an arbitrary small amount of time. We apply the basic principles of the method on the linear motorcycle model described by equations (1-2) to obtained the transfer function $H(s)$ between the output $\dot{\psi}$ and the input $\delta$ :

$$
H=\frac{Y}{U}=\frac{N_{3} s^{3}+N_{2} s^{2}+N_{1} s+N_{0}}{s^{4}+D_{3} s^{3}+D_{2} s^{2}+D_{1} s+D_{0}}
$$

the coefficients $N_{0}, . ., N_{3}, D_{0}, . ., D_{3}$ are non-linear functions of the parameters $I_{x}$ and $I_{z}$ (the inertia parameters $I_{x}$ and $I_{z}$ are unknown, to be identified from the transfer function).

If we can identify the coefficients of the transfer function, we can deduce the values of the system parameters by a set of equations. We can rewrite the transfer function from equation (7) into the following form:

$y^{(4)}+D_{3} y^{(3)}+D_{2} \ddot{y}+D_{1} \dot{y}+D_{0} y=N_{3} u^{(3)}+N_{2} \ddot{u}+N_{1} \dot{u}+N_{0} u$

where the exponent $(i)$ indicates the derivation of order $i$ with respect to $t$.

We proceed to compute the unknowns system parameters $D_{i}$, $N_{i}$ as follows: In the Laplace domain, the time derivative is equivalent to the product $s$. Taking laplace transform of the expression 8 and by multiplying $s$ :

$$
\begin{aligned}
& {\left[s^{5} y(s)-s^{4} y(0)-s^{3} y(0)-s^{2} y(0)-s y(0)\right]} \\
& +D_{3}\left[s^{4} y(s)-s^{3} y(0)-s^{2} y(0)-s y(0)\right]+\ldots+D_{0} s y(s) \\
& =N_{3}\left[s^{4} u(s)-s^{3} u(0)-s^{2} u(0)-s u(0)\right]+\ldots+N_{0} s u
\end{aligned}
$$

Since the initial conditions are unknown so we differentiate five times so that all constant terms leave, then :

$$
\begin{aligned}
& \frac{\partial^{5}}{\partial s^{5}}\left[s^{5} y\right]+D_{3} \frac{\partial^{5}}{\partial s^{5}}\left[s^{4} y\right]+D_{2} \frac{\partial^{5}}{\partial s^{5}}\left[s^{3} y\right]+D_{1} \frac{\partial^{5}}{\partial s^{5}}\left[s^{2} y\right]+ \\
& D_{0} \frac{\partial^{5}}{\partial \partial^{5}}[s y]=N_{3} \frac{\partial^{5}}{\partial s^{5}}\left[s^{4} u\right]+N_{2} \frac{\partial^{5}}{\partial s^{5}}\left[s^{3} u\right]+ \\
& N_{1} \frac{\partial^{5}}{\partial s^{5}}\left[s^{2} u\right]+N_{0} \frac{\partial^{5}}{\partial s^{5}}[s u]
\end{aligned}
$$

Then we develop each term of (10), Let's calculate $\frac{\partial^{5}}{\partial s^{5}}\left[s^{5} y\right]$

$$
\begin{aligned}
\frac{\partial^{5}}{\partial s^{5}}\left[s^{5} y\right]= & 5 ! y+5 ! \times C_{5}^{1} \times s \frac{\partial y}{\partial s}+\frac{5 !}{2 !} \times C_{5}^{2} \times s^{2} \frac{\partial^{2} y}{\partial s^{2}} \\
& +\frac{5 !}{3 !} \times C_{5}^{3} \times s^{3} \frac{\partial^{3} y}{\partial s^{3}}+\frac{5 !}{4 !} \times C_{5}^{4} \times s^{4} \frac{\partial^{4} y}{\partial s^{4}} \\
& +\frac{5 !}{5 !} \times C_{5}^{5} \times s^{5} \frac{\partial^{5} y}{\partial s^{5}}
\end{aligned}
$$

with $C_{n}^{r}=\frac{n !}{r !(n-r) !}$, by the same way, $\frac{\partial^{5}}{\partial s^{5}}\left[s^{4} y\right]$ we have :

$$
\begin{aligned}
\frac{\partial^{5}}{\partial s^{5}}\left[s^{4} y\right]= & 4 ! \times C_{5}^{1} \times \frac{\partial y}{\partial s}+4 ! \times C_{5}^{2} \times s \frac{\partial^{2} y}{\partial s^{2}} \\
& +\frac{4 !}{2 !} \times C_{5}^{3} \times s^{2} \frac{\partial^{3} y}{\partial s^{2}}+\frac{4 !}{3 !} \times C_{5}^{4} \times s^{3} \frac{\partial^{4} y}{\partial s^{4}} \\
& +\frac{4 !}{4 !} \times C_{5}^{5} \times s^{4} \frac{\partial^{5} y}{\partial s^{5}}
\end{aligned}
$$

The other terms are calculated using the same procedure.

Since it is already known, from the theory of the Laplace transform, multiplication by $s$ means a derivation with respect to $t$ in time domain, which is not a numerically robust operation. This is why we multiply the equation by $s^{-5}$ to avoid unnecessary derivations.

we calculate $s^{-5} \frac{\partial^{5}}{\partial s^{5}}\left[s^{5} y\right]$

$$
\begin{array}{r}
s^{-5} \frac{\partial^{5}}{\partial s^{5}}\left[s^{5} y\right]=5 ! s^{-5} y+5 ! \times C_{5}^{1} \times s^{-4} \frac{\partial y}{\partial s}+\frac{5 !}{2 !} \times C_{5}^{2} \times s^{-3} \frac{\partial^{2} y}{\partial s^{2}} \\
+\frac{5 !}{3 !} \times C_{5}^{3} \times s^{-2} \frac{\partial^{3} y}{\partial s^{2}}+\frac{5 !}{4 !} \times C_{5}^{4} \times s^{-1} \frac{\partial^{4} y}{\partial s^{3}}+\frac{5 !}{5 !} \times C_{5}^{5} \times \frac{\partial^{5} y}{\partial s^{4}} \\
s^{-5} \frac{\partial^{5}}{\partial s^{5}}\left[s^{4} y\right]=4 ! \times C_{5}^{1} \times s^{-5} \frac{\partial y}{\partial s}+4 ! \times C_{5}^{2} \times s^{-4} \frac{\partial^{2} y}{\partial s^{2}}+ \\
\frac{4 !}{2 !} \times C_{5}^{3} \times s^{-3} \frac{\partial^{3} y}{\partial s^{2}}+\frac{4 !}{3 !} \times C_{5}^{4} \times s^{-2} \frac{\partial^{4} y}{\partial s^{4}}+\frac{4 !}{4 !} \times C_{5}^{5} \times s^{-1} \frac{\partial^{5} y}{\partial s^{5}}
\end{array}
$$

and so on for the other terms. Thus, in the temporal domain, the resulting equation can be written as

$$
\begin{aligned}
& D_{3} p_{1 \_1}+D_{2} p_{1 \_2}+D_{1} p_{1 \_3}+D_{0} p_{1 \_4}+N_{3} p_{1 \_5} \\
& +N_{2} p_{1 \_}+N_{1} p_{1 \_}+N_{0} p_{1 \_}=-q_{1}
\end{aligned}
$$

$q_{1}$ represents the temporal form of the equation $s^{-5} \frac{\partial^{5}}{\partial s^{5}}\left[s^{5} y\right]$

$$
\begin{aligned}
& q_{1}=-5 ! C_{5}^{1} \int^{5} t y+5 ! C_{5}^{2} \int^{4} t^{2} y-\frac{5 !}{2 !} C_{5}^{3} \int^{3} t^{3} y+\frac{5 !}{3 !} C_{5}^{4} \int^{2} t^{4} y \\
& -\frac{5 !}{4 !} C_{7}^{5} \int^{5} y+\frac{5 !}{5 !} C_{5}^{5} \int^{2} t^{5} y-\frac{5 !}{5 !} C_{5}^{5} \int t^{7} y
\end{aligned}
$$

The expressions of $p_{1 \_1}, p_{1 \_2}, \ldots, p_{1 \_8}$ and $q_{1}$ are written as a differential equation.

The matrix can be completed by integrating the previous equation and making it invertible. Then we can deduce a matrix relation. The coefficients are identified by solving equation (13).

$$
\underbrace{\left(\begin{array}{cccc}
p_{1 \_1} & p_{1 \_2} & \ldots & p_{1 \_8} \\
p_{2 \_1} & p_{2 \_} 2 & \ldots & p_{2 \_} \\
\vdots & \vdots & \ddots & \vdots \\
p_{8 \_1} & p_{10 \_2} & \ldots & p_{8 \_8}
\end{array}\right)}_{\mathscr{P}}\left(\begin{array}{c}
D_{3} \\
\vdots \\
D_{0} \\
N_{3} \\
\vdots \\
N_{0}
\end{array}\right)=\underbrace{-\left(\begin{array}{c}
q_{1} \\
q_{2} \\
\vdots \\
q_{8}
\end{array}\right)}_{\mathscr{Q}}
$$

with $p_{8 \_i}=\int p_{7_{-} i}=\iint p_{6 \_i}=\iiint p_{5_{-} i}=\ldots=$ $\int^{7} p_{1 \_i}$ pour $\forall i=\{1,2, \ldots, 8$. 
and $\quad q_{8}=\int q_{7}=\iint q_{6}=\iiint q_{6}=\ldots=\int^{7} q_{1}$ pour $\forall i=$ $\{1,2, \ldots, 8\}$

Implementation point of view : each component of the $\mathscr{P}$ and $\mathscr{Q}$ matrix can be written in a state space framework by a simple linear variant time filter. The excitation input plays a very important role in the identification of the transfer function.

\section{SIMULATION RESULT}

\section{A. Static Test : geometric parameters $a, b, h$ and $m$}

As previously seen, the center of mass of a motorcycle is defined by the values of the parameters $a, b$ and $h$ for a one-body model. This point can vary with the weight of the rider. Thus, we distinguished two different cases (with and without rider), we used the static method presented in section (3), each scenario being tested once without the rider then with rider.

With the measurements of the vertical forces it is possible to calculate the values of the parameters $a, b$ and $h$ by equation (5).

Table I presents experimental test carried out on the scooter of the laboratory .

TABLE I

GEOMETRIC PARAMETERS $a, b, h(m), m(k g)$

\begin{tabular}{|l|l|l|l|l|}
\hline geomtric parameters & (a) & (b) & (h) & (m) \\
\hline motorcycle without rider & 0.7363 & 0.5637 & 0.3960 & 142 \\
\hline motorcycle with rider & 0.8015 & 0.4985 & 0.5194 & 221 \\
\hline
\end{tabular}

First, the rider influence is clearly checked, especially for the $h$ parameter. The values of $a$ and $b$ share the wheelbase with a ratio of (57\% and $43 \%)$ without rider, $(62 \%$ and $38 \%$ ) with rider.

\section{B. Identification of inertial parameters}

From differential model, we realized a motorcycle model of a rigid body taking the values of geometric parameters calculated from the static test, for the pneumatic parameters that are influenced by tire types and road condition, we consider the typical values presented in [3] and the values of other accessible parameters are taken from data sheet of the motorcycle. By the nature of the system, the model is unstable. It was necessary to add a controler to guarantee the stability of the system with state feedback computed by poles placement (to replacement rider). The dynamics of the motorcycle has been analyzed with a Sharp model [1] existing in the database of the simulation software "Bikesim", this bike is similar to the scooter in terms of dynamics and characteristic. Bikesim offers the possibility to create movements using excitation inputs: steering angle, acceleration, braking, and taking into account the climatic parameters such as wind. The excitation signal plays a very important role in parameters identification, so we have chosen the excitation input using Bikesim so that the angular accelerations will be well excited without falling the motorcycle.

\section{Analysis of the dynamic behavior}

The results presented below allow to study the behavior of the motorcycle identical to that used for identification, combining braking in a straight line and a steering wheel action, to allow us choosing the best input excitation.

1) Braking in a straight line: Straight-line braking tests show mainly the longitudinal behavior along $X_{G}$ and the pitching behavior around the $Y_{G}$ axis [17].

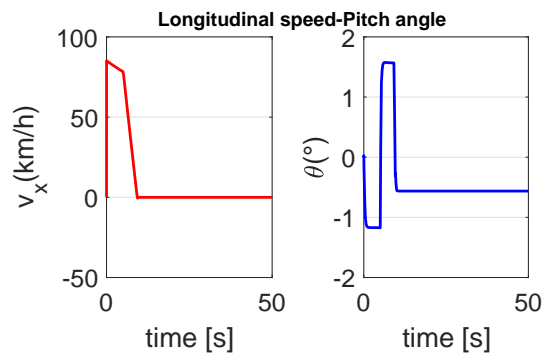

2) Step Steer: The stepping-wheel steering - sudden steering of the stepped wheels Fig. 3 - allows to study the behavior of the vehicle in the transverse direction $Y_{G}$ and in roll - rotation around the axis $X_{G}$.
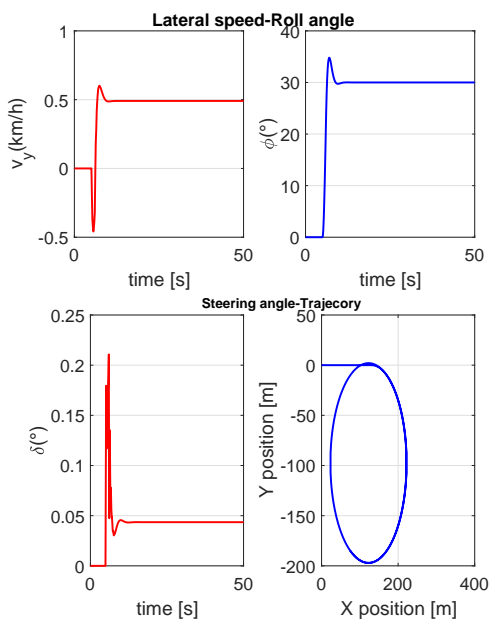

Fig. 3. Lateral speed-Roll angle-Step Steer-Trajectory.

3) Flying sinus: the steering angle follows a law of the wobbled sinus type, the flying angle (Figure 5) is of sinusoidal shape but of progressively increasing frequency and amplitude progressively decreasing. These tests involve the two-wheeled vehicle mainly in yaw and in rolling which allows us to identify the associated modes : 


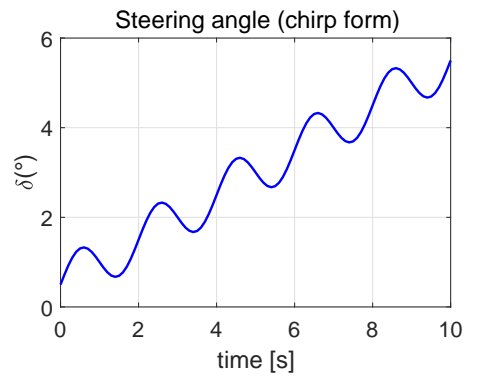

Fig. 4. Steering angle $\delta$ : flying angle

\section{Algebraic identification}

The study of transfer functions of type: (yaw rate $\dot{\psi}$ /steering angle $\delta$ ) allows us to identify the inertial parameters of the motorcycle body. We have excited the dynamics with a flying sinus in the steering angle, then measurement data are used in the parametric identification method to obtain the following transfer function:

$$
\begin{gathered}
H_{i d}(s)=\frac{\dot{\psi}}{\delta}=\frac{503.9 s^{3}+1.511 \times 10^{4} s^{2}-9.22 \times 10^{4} s-1.317 \times 10^{6}}{s^{4}+158.5 s^{3}+5749 s^{2}+4.136 \times 10^{4} s+4.755 \times 10^{4}} \\
=\frac{N_{3 i d} s^{3}+N_{2 i d} s^{2}+N_{1 i d} s+N_{0 i d}}{s^{4}+D_{3 i d} s^{3}+D_{2 i d} s^{2}+D_{1 i d} s+D_{0 i d}}
\end{gathered}
$$

The coefficients of the transfer function calculated from the state space model (7) of the motorcycle: $\left[D_{i}, N_{i}\right]$ are functions of the unknown parameters $I_{x}, I_{z}$, they are highly complex and non-linear, after simplification, we get the denominator coefficient:

$D_{3}=\frac{3.5837 \times 10^{3}}{I_{z}}+\frac{528.1462}{I_{x}}+13.1750$

$D_{2}=\frac{6.0275 \times 10^{4}}{I_{z}}+\frac{1.4796 \times 10^{3}}{I_{x}}+2.0384 \times 10^{6}$

$D_{1}=\frac{2.3268 \times 10^{7}}{I_{x} \cdot I_{z}}-\frac{2.0230 \times 10^{4}}{I_{z}}$

$D_{0}=\frac{2.6024 \times 10^{7}}{I_{x} \cdot I_{7}}$ we have to find two parameters $I_{x}$ and $I_{z}$, we show only the two coefficients $D_{3}$ and $D_{2}$ of the denominator :
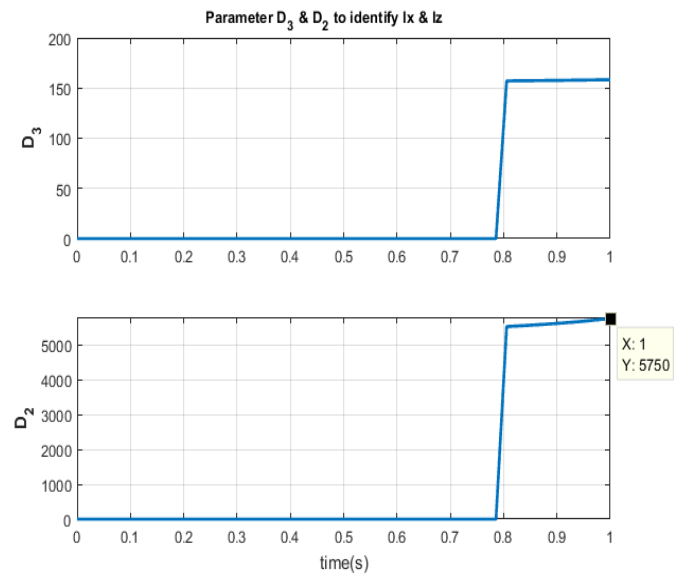

Fig. 5. Convergence

It can be seen, from the above simulation that the method converges after $0.8(s)$ this is due to the response time of the filters

After estimation by the algebraic method we calculate the values of parameters $I_{x}$ and $I_{z}$ from the expressions of $D_{3}$ and $D_{2}$ and the identified function $H_{i d}$ :

$$
I_{x_{\text {estim }}}=17.609 \mathrm{k}_{\mathrm{g}} \cdot \mathrm{m}^{2}, I_{z_{\text {estim }}}=31.073 \mathrm{~kg}_{\mathrm{g}} \cdot \mathrm{m}^{2}
$$

Note that the prior inertial parameters are computed from BIKESIM using the huygens theorem :

$$
I_{x_{\text {bikesim }}}=17.623 \mathrm{~kg}_{\mathrm{g}} \mathrm{m}^{2}, I_{z_{\text {bikesim }}}=31.058 \mathrm{~kg}_{\mathrm{g}} \cdot \mathrm{m}^{2}
$$

\section{E. Model validation with data measurement}

We had performed test scenarios with a scooter motorcycle, instrumented with several sensors (odometer, GPS sensor, IMU..). After that, we have analyzed and processed data obtained by rancing scenarios, in order to find consistent results compared to real situation. To validate the model, it

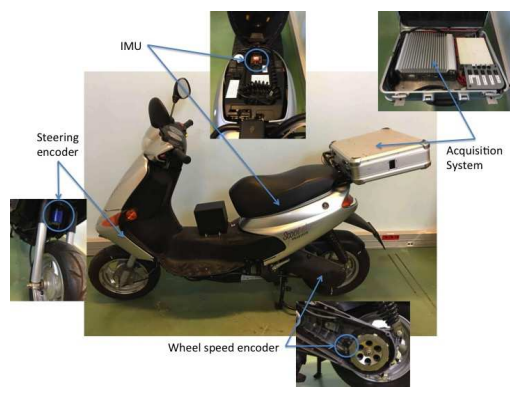

Fig. 6. scooter

is sufficient to recover the input data acquired during the experiment for exciting the identified model and observe the output of the model, thus comparing it with the measured output.

From experiments conducted, a scenario is chosen where the motorcycle was turning on a mini-roundabout to better check the variation of the roll angle which is given by the IMU (10 DoF ) placed at the center of gravity under the seat.

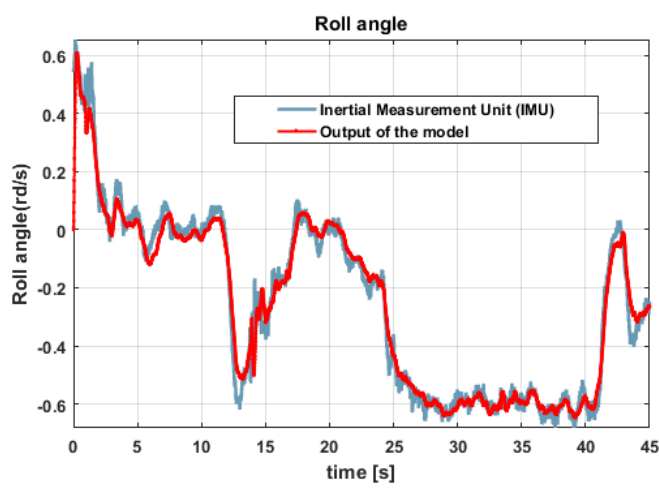

Fig. 7. Validation of the model: turning at the roundabout

The graphs (Figure 7) show that the output of the model is coherent with the measurements of the experiments. The measurement are slightly disturbed, the measured roll angle of and model have a very close profiles, the simulation results converge well to the data measured by sensors embedded on the motorcycle. 


\section{CONCLUSIONS}

This paper presents an algebraic identification approach, used to identify the center of gravity and the inertial parameters of a powered two wheels vehicle modeled as a rigid body.

The difficulties encountered concern the choice of the persistent input to excite the dynamics of the motorcycle (effect of bad conditionings).

The model is validated by the data measurement carried out on a scooter. In future works, it is interesting to identify a non-linear multi-body model of motorcycles which includes additional parameters and construct a robust motorcycle control law with respect to parametric uncertainties. Another perspective is to take into account the geometry of the road which has been considered flat and to consider the effect of the rider in the dynamics.

\section{APPENDIX}

The system state matrices are :

$M=\left(\begin{array}{cccccc}0 & -m v_{x} & 0 & 0 & 1 & 1 \\ 0 & 0 & 0 & 0 & a & -b \\ 0 & 0 & 0 & 1 & 0 & 0 \\ 0 & m h v_{x} & m g h & 0 & 0 & 0 \\ -\frac{C_{f 1}}{v_{x}} & \frac{a C_{f 1}}{v_{x}} & C_{f 2} & 0 & -\frac{v_{x}}{\tau} & 0 \\ -\frac{C_{r 1}}{v_{x}} & \frac{b C_{r 1}}{v_{x}} & C_{r 2} & 0 & 0 & -\frac{v_{x}}{\tau}\end{array}\right) N=\left(\begin{array}{c}0 \\ 0 \\ 0 \\ 0 \\ C_{f 1} \\ 0\end{array}\right)$

$E=\left(\begin{array}{cccccc}m & 0 & 0 & 0 & 0 & 0 \\ 0 & I_{z} & 0 & 0 & 0 & 0 \\ 0 & 0 & 1 & 0 & 0 & 0 \\ -m h & 0 & 0 & I_{x} & 0 & 0 \\ 0 & 0 & 0 & 0 & 1 & 0 \\ 0 & 0 & 0 & 0 & 0 & 1\end{array}\right) F\left(v_{x}(t)\right)=\left(\begin{array}{cc}0 & 0 \\ 0 & 0 \\ 0 & 0 \\ 0 & 0 \\ \frac{v_{x}}{\tau} & 0 \\ 0 & \frac{v_{x}}{\tau}\end{array}\right)$

$C=\left(\begin{array}{cccccc}0 & 1 & 0 & 0 & 0 & 0 \\ 0 & 0 & 1 & 0 & 0 & 0 \\ 0 & 0 & 0 & 1 & 0 & 0 \\ 0 & 0 & 0 & 0 & \frac{1}{m} & \frac{1}{m}\end{array}\right), \quad$ and $u(t)=\delta, d(t)=\left(\begin{array}{c}\Delta F_{y f}^{S} \\ \Delta F_{y r}^{S}\end{array}\right)$

- $[a, b, h]$ : geometric variables.

- $[m]$ : motorcycle mass.

- $[g]$ : acceleration gravity.

- $\left[v_{x}, v_{y}\right]$ : longitudinal and lateral velocity.

- $\left[I_{x}, I_{z}\right]$ : moment of inertia along the $x$ and $z$ axes.

- $[\phi, \psi]$ : roll and the yaw angle.

- $[\boldsymbol{\delta}]$ : steering angle imposed by the rider

- $\left[G_{t}\right]$ : center of gravity of the vehicle.

- $\left[F_{y f}, F_{y r}\right]$ : the lateral force applied to the front and the rear wheel.

- $\left[C_{r 1}, C_{r 2}, C_{f 1}, C_{f 2}\right]$ : pneumatic coefficients (r: rear wheel, $\mathrm{f}$ : front wheel, 1: stiffness of the slide, 2 camber stiffness)

- $\left[\Delta F_{y r}^{S}, \Delta F_{y f}^{S}\right]$ : nonlinear part.

\section{REFERENCES}

[1] Sharp, Robin S, "The stability and control of motorcycles", Journal of mechanical engineering science, vol. 13,pp 316-329, 1971.

[2] Nishimi, Tomoo and Aoki, Akira and Katayama, Tsuyoshi Nishimi, T., Aoki, A., Katayama, T. (1985). Analysis of straight running stability of motorcycles (No. 856124). SAE Technical Paper.
[3] Ichalal, D., Dabladji, H., Arioui, H., Mammar, S., Nehaoua, L. (2013, June). Observer design for motorcycle lean and steering dynamics estimation: a takagi-sugeno approach. In American Control Conference (ACC), 2013 (pp. 5654-5659). IEEE.

[4] Slimi, H., Arioui, H., Nouveliere, L., Mammar, S. (2010, June). Motorcycle speed profile in cornering situation. In American Control Conference (ACC), 2010 (pp. 1172-1177). IEEE.

[5] Arioui, H., Hima, S., Nehaoua, L. (2009, July). 2 DOF low cost platform for driving simulator: Modeling and control. In Advanced Intelligent Mechatronics, 2009. AIM 2009. IEEE/ASME International Conference on (pp. 1206-1211). IEEE.

[6] James, S. R. (2002). Lateral dynamics of an offroad motorcycle by system identification. Vehicle System Dynamics, 38(1), 1-22.

[7] Tanelli, M., Corno, M., Saveresi, S. (2014). Modelling, simulation and control of two-wheeled vehicles. John Wiley Sons.

[8] Pradeepak, R., Bhambri, M., Rahman, S. (2015). A Parametric Multi Body Approach to Find the Key Elements Influencing the Steering Torque Applied by Rider on a Motorcycle (No. 2015-01-0641). SAE Technical Paper.

[9] Fliess, M., SiraRamrez, H. (2003). An algebraic framework for linear identification. ESAIM: Control, Optimisation and Calculus of Variations, 9, 151-168.

[10] Mamani, G., Becedas, J., Feliu-Batlle, V., Sira-Ramirez, H. (2007, July). Open-loop algebraic identification method for a DC motor. In Control Conference (ECC), 2007 European (pp. 3430-3436). IEEE.

[11] Becedas, J., Trapero, J. R., Sira-Ramirez, H., Feliu-Battle, V. (2007, April). Fast identification method to control a flexible manipulator with parameter uncertainties. In Robotics and Automation, 2007 IEEE International Conference on (pp. 3445-3450). IEEE.

[12] Becedas, J., Mamani, G., Feliu-Batlle, V., Sira-Ramirez, H. (2007, October). Algebraic identification method for mass-spring-damper system. In WCECS 2007, Proceedings of the World Congress on Engineering and Computer Science 2007.

[13] Nehaoua, L., Arioui, H., Seguy, N., Mammar, S. (2013). Dynamic modelling of a two-wheeled vehicle: Jourdain formalism. Vehicle System Dynamics, 51(5), 648-670.

[14] Reger, J., Jouffroy, J. (2009, December). On algebraic time-derivative estimation and deadbeat state reconstruction. In Decision and Control, 2009 held jointly with the 2009 28th Chinese Control Conference. CDC/CCC 2009. Proceedings of the 48th IEEE Conference on (pp. 1740-1745). IEEE.

[15] Baronti, F., Zamboni, W., Femia, N., Rahimi-Eichi, H., Roncella, R., Rosi, S., Chow, M. Y. (2013, May). Parameter identification of LiPo batteries in electric vehicles: A comparative study. In Industrial Electronics (ISIE), 2013 IEEE International Symposium on (pp. 1-7). IEEE.

] Neves, A. (2005). Identification Algbrique et Dterministe de Signaux et Systmes Temps Continu: Application des Problmes de Communication Numrique (Doctoral dissertation, Universit Ren Descartes-Paris V).

[17] Dabladji, M. E. H., Ichalal, D., Arioui, H., Mammar, S. (2017). Toward a Robust Motorcycle Braking. IEEE Transactions on Control Systems Technology, 25(3), 1052-1059. 